\title{
Role of physical activity in the occurrence of falls and fall-related injuries in community-dwelling adults over 50 years old
}

\author{
Catarina L. N. Pereira ${ }^{1}$, Fátima Baptista ${ }^{2}$, and Paulo Infante ${ }^{3}$ \\ ${ }^{1}$ Department of Sports and Health, University of Évora, Évora, Portugal, ${ }^{2}$ Exercise and Health Laboratory, Faculty of Human Movement, \\ Technical University of Lisbon, Portugal, and ${ }^{3}$ Department of Mathematics, University of Évora, Évora, Portugal
}

\section{Abstract}

Purpose: This study examined the effect of the type, level and amount of physical activity in falls and fall-related injuries. Method: Participants were 506 community-dwelling adults aged $>50$ years (390 women: $67.7 \pm 6.8$ years and 116 men: $69.6 \pm 6.6$ years). Falls, fall-related injuries (slight and severe), and physical activity (type, level and energy expenditure) were evaluated by questionnaires. Confounders included co-morbidities, fear of falling, environmental hazards and physical fitness. Results: After adjustment for confounders, logistic analysis revealed that the likelihood of falling decreased by $2 \%$ for each 100 metabolic expenditure (MET-min/week) of total physical activity and increased by $5 \%$ for each 100 MET-min/week of vigorous-intensity physical activity; total physical activity $>1125$ MET-min/week and vigorous physical activity $<500 \mathrm{MET}$-min/week were identified as cut-off values discriminating non-fallers from fallers. Compared to the low physical activity level, increased physical activity levels diminished the likelihood of the occurrence of severe fall-related injuries by $76 \%$ (moderate) and $58 \%$ (high; $p<0.05$ ) in fallers. Conclusions: Being active, especially sufficiently active, reduces fall-related injuries by decreasing falls and by safeguarding against severe injuries when falls occur. At least $1125 \mathrm{MET}-\mathrm{min} /$ week of total physical activity including $>500 \mathrm{MET}-$ min/week of vigorous intensity seems to prevent falls and, therefore, fall-related injuries.

\section{> Implications for Rehabilitation}

- Being sufficiently active reduces fall-related injuries by reducing falls and by safeguarding against severe injury when falls occur

- For each additional amount of total physical activity there is a corresponding direct (due to the effect of isolated physical actvivity) and indirect (due to the subject gaining in fitness) decrease in the risk of falling and thus injury. Vigorous physical activity leads to an increase in total physical activity; however, it also leads to an increase in the risk of falling and injury

- Total physical activity of at least 1125 MET-min/week with equal or lower than 500 MET-min/ Keywords

Community-dwelling adults, environmental hazards, fall-related injuries, falls, physical activity

\section{History}

Received 31 March 2012

Revised 16 February 2013

Accepted 1 March 2013

Published online 1 ] week (i.e. less than $\sim 1 \mathrm{~h}$ /week, according IPAQ criteria) of vigorous intensity significantly reduces falls and therefore injury

\section{Introduction}

A fall is an unexpected event in which a person suddenly and involuntary comes to rest on the ground, floor or lower level [1] that usually occurs when the demands of a given task exceed the person's ability. The demands of a task are determined by its difficulty and by environmental conditions [2-4]. The ability to perform daily tasks without falling declines with age due to diminishing physical, sensorial and mental functions; moreover, a weak health status also intensifies this process [5,6]. These facts, combined with increasing life expectancy [7], have led to an increase in the incidence of falls and their consequences in older populations [8]. However, falls may

Address for correspondence: Catarina L. N. Pereira, Department of Sports and Health, University of Évora, Pavilhão Gimnodesportivo, Rua 65 Reguengos de Monsaraz, 44, 7000-727 Évora, Portugal. Tel: +351 266 769 522. Fax: +351 266769 521. E-mail: clnp@uevora.pt also be a problem before the age of 60 [9], despite the fact that the risk profile for the occurrence of falls may differ between middleaged and elderly people [10]. The main immediate consequence of a fall is injury, which may sometimes be fatal. Another possible shortterm consequence is an increased fear of falling that often leads to a loss of functional independence while performing daily tasks $[11,12]$.

Physical activity is a key factor for the maintenance of healthrelated quality of life, including functional competence [13]. However, the performance of any physical task, including daily tasks, is inevitably associated with a certain risk of falling and injury. For this reason, many community-dwelling older people and some carers might believe that inactivity is the best way to eliminate or reduce that risk [14].

Effective physical activity/exercise reduces the rate of falling in older people by $17 \%$ [15]. However, not only a low level, but also a high level of physical activity has been associated with an 
increased fall risk [16,17], with regard to the exposure to hazards involved $[1,14]$. Besides this, it is known that the risk of falling varies according to the type of activities [18], as does the risk of fall-related injury [19]. It is not clear, however, what amount or intensity of physical activity/exercise is needed to reduce the occurrence of falls and related injuries or how physical activity interacts with other risk factors for falls. The successful completion of daily tasks (without falling) is dependent on intrinsic factors such as age, health, physical fitness and fear of falling, and also on extrinsic factors such as the demands of each task and environmental hazards $[4,20,21]$. Thus the main purpose of this study was to analyse the role of physical activity in falls and related injuries in community-dwelling adults aged over 50 years, adjusting for these intrinsic and extrinsic risk factors.

\section{Method}

\section{Subjects}

Participants were 506 community-residing adults aged over 50 years (390 women: $67.7 \pm 6.8$ years and 116 men: $69.6 \pm 6.6$ years), of which $31 \%$ had fallen at least once in the previous 12 months, and $78 \%$ were attending a supervised exercise program at least twice per week. Exercise programs consist of single or combined activities such as hydrogymnastics, aerobics and step, swimming, yoga, tai chi, weight training, cycling, dancing, tennis and golf. Participants were recruited by invitation and via leaflets and posters distributed in community settings (health centres, recreational, sporting, and cultural organisations, senior universities, etc.) and included 153 middle-aged persons $(<65$ years) and 353 aged persons $(\geq 65$ years). All subjects lived independently and did not suffer from recent illnesses or disabilities resulting in a temporary loss of physical function. Inclusion in the study required the absence of cognitive impairment according to the criteria established by the Folstein Mini-Mental State Examination [22]. A single interviewer filled out evaluation questionnaires and verified the inclusion criteria. Forty-one volunteers did not meet these criteria and were excluded, including two subjects who had experienced hip fractures due to a fall in the previous months, due to their physical function being affected. All subjects were volunteers, and provided informed consent. The university's temporarily ethics committee approved this study.

\section{Measures and data collection}

Physical activity

Physical activity was assessed using the short form of the International Physical Actiyity Questionnaire (IPAQ) [23]. This covers metabolic expenditure (MET-min/week) in three types of physical activity: walking (3.3 MET), moderate (4.0 MET), and vigorous-intensity activity (8.0 MET). Metabolic expenditure was calculated by determining the time $(\mathrm{min} / \mathrm{d})$ and frequency $(\mathrm{d} /$ week) spent on each type of physical activity. For example, walking MET-min/week was calculated as: $3.3 \times$ walking $\min \times$ walking d. Total physical activity was the sum of all metabolic expenditure. Individuals were also categorised as "low," "moderate" or "high active" level according to the IPAQ criteria [23]. In order to ensure a representative measure of habitual physical activity, the interviewer asked every participant to relate their activity during a typical week.

\section{Falls and fall-related injuries}

The number of falls in the previous 12 months, the circumstances surrounding each fall and its consequences were assessed using a questionnaire administered by an interviewer.
In order to enhance statistical power (by increasing the number of analysed subjects), a faller was defined as a subject who had fallen at least once in the previous 12 months and not only as a subject presenting recurrent falls [24]. The number of falls was categorised as absence of fall, one fall, two falls, three falls and four or more falls. Injury severity was classified as slight (no injury, slight scratches and oedema) and severe (serious abrasion, strained muscles, torn muscles, sprains, dislocations and fractures). For the statistical analysis of injuries, it was decided to consider each person's most severe fall (the fall that resulted in the most severe injury).

\section{Potential confounders}

Physical fitness. Lower and upper body strength and flexibility, agility, aerobic endurance and body mass index were evaluated by using the Fullerton functional fitness test battery [25], namely 30 s chair stand (repetitions or reps in $30 \mathrm{~s}$ ); $\operatorname{arm}$ curl (reps in $30 \mathrm{~s}$ ); chair sit-and-reach $(\mathrm{cm})$; back scratch $(\mathrm{cm}) ; 8 \mathrm{ft}$ up-and-go $(\mathrm{s})$; 6 min walk (m). Mean fitness (SD) was estimated as the average of the standardised scores (Z-score) achieved in each test of the battery (excluding body mass index) [26]. Standing height $(\mathrm{cm})$ was measured with a stadiometer (Secca 770, Hamburg, Germany), body weight was determined using an electronic scale (Secca Bella 840, Hamburg, Germany) and body mass index $\left(\mathrm{kg} / \mathrm{m}^{2}\right)$ was calculated.

Balance was evaluated using the Fullerton advanced balance (FAB) scale [27]. This includes the following tests: stand with feet together, eyes closed; reach forward to object; turn full circle; step up and over, tandem walk; stand on one leg; stand on foam, eyes closed; two-footed jump; walk with head turns and reactive postural control. The final score was calculated as the sum of points obtained in each of the 10 tests, from 0 (worst) to 4 (best), with a total range of $0-40$ points.

Additionally, grip strength (bars) was measured using a pressure infusion hand dynamometer (Dynatest Riester) in a standing position, with the elbow extended, being considered the best result of two trials performed by the dominant hand; waist circumference $(\mathrm{cm})$ was measured at the level of the navel using a tape measure, and fat body mass (\%) was evaluated by means of bio-impedance [28] (HBF-306C, Schaumburg, IL). Lean body mass index $\left(\mathrm{kg} / \mathrm{m}^{2}\right)$ was calculated as [total body mass $(\mathrm{kg})$-fat body mass $(\mathrm{kg})] /\left[\right.$ body height $\left.{ }^{2}\left(\mathrm{~m}^{2}\right)\right]$.

Fear of falling. Fear of falling was assessed using the modified version of the Falls Efficacy scale (FES) [29,30]. All participants reported how concerned they felt about falling while performing each of the 10 daily activities listed in FES. Each item was rated on a points scale from 0 (not concerned) to 3 (very concerned). The total score was the sum of the points obtained in each of the tests for a range from 0 to 30 .

\section{Environmental hazards, co-morbidities and education}

The quantification of environmental hazards, diseases, physical impairments and educational level were assessed using the questionnaire administered by the interviewer.

All chronic diseases reported by participants were listed (totalling 24). Physical impairments included involuntary loss of urine, frequent dizziness, foot problems (e.g. sores, corns, skewed toes, amputation of toes or foot, insufficient muscle function), poor vision (not recognising someone's face at a distance of $4 \mathrm{~m}$ with glasses or contact lenses), hearing problems (not able to follow a conversation in a group of four people with a hearing aid) and balance problems (occasional loss of balance) [30]. The presence or absence of each disease and physical impairment was 
checked for each participant. The number of chronic diseases and physical impairments defined co-morbidities.

Environmental hazards included: indoor hazards (bad lighting, slippery floors, loose rugs, telephone cables, other objects, ladders, stairways with steep steps, without walls and/or handrails, kitchens with difficult access to utensils and movable tables, bathrooms without tub handrails, shower and toilet and non-skid mat in tub or shower, bed too high or too low), outdoor hazards (bad lighting, uneven pavements, streets, paths, repair works, obstacles, slippery floors), the presence of animals and footwear. For each participant the presence or absence of each listed environmental hazard was checked and the total number of hazards counted (minimum: 0, maximum: 34) [31].

\section{Statistical analysis}

Participants' characteristics (age, co-morbidities, fear of falling, hazard environments, physical activity/inactivity, and physical fitness) were compared between groups (fallers versus non-fallers; and fallers with severe injuries versus fallers without severe injuries) by using independent $t$-tests, after the verification of data distribution assumptions. Comparisons of the prevalence of physical activity level and gender between these groups were made using Chi-Square Pearson's test or Fisher's test.

Binary logistic regressions [32] were used to identify predictor variables of falls and related severe injuries. In order to include all significant variables in the multivariate analysis, the most parsimonious model was determined. The Wald statistic was used to test the significance of each variable and Likelihood Ratio was calculated in order to compare each new model with the previous one. The assumption of linearity in continuous variables was checked using the logit function and the overall fit was evaluated using the Hosmer-Lemeshow goodness-of-fit test. Whenever correlations between variables were above 0.8 , only the most explanatory variable was retained in the model. Outliers and influential points were identified. Receiver operating characteristic (ROC) analysis was used to examine the discriminative ability of the models used to predict falls and injuries. A cut-off point for falls probability was established by maximising both sensitivity and specificity [33]. This cut-off point was then used to identify the amount of physical activity (cut-off values) that discriminates fallers from non-fallers by solving the equation 331 resulting from binary logistic regression. Internal validation of the 332 model was performed by re-sampling or cross-validation proced- 333 ure [34]. For cross-validation, participants were divided into 10334 equal groups by sampling randomly without replacement. $\quad 335$

In order to identify possible cut-off values that might affect 336 results from statistical analysis, age, the number of falls and injury 337 severity were classified and categorised into different forms. The 338 significance of each new variable was studied in the regression 339 models and the best classification/categorisation was selected. 340 Physical activity values were presented as 100 MET-min/week in 341 order to interpret the data from regression analysis.

Complementary analysis was performed using the Pearson or 343 Spearman correlation tests and Poison regression. Statistical 344 analyses were performed with SPSS version 17.0, considering 345 statistical significance to be $p \leq 0.05$.

\section{Results}

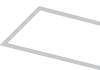

One hundred and fifty-eight participants $(31.2 \%)$ had fallen in the previous 12 months, giving a total of 298 falls. No differences were found between middle-age and aged participants concerning the amount of physical activity, but the proportion of indoor falls was higher than outdoor falls in middle-aged people (indoor: 51.9\% versus outdoor: $48.1 \%$ ) while the proportion of outdoor falls was higher in aged people (indoor: $40.2 \%$ versus outdoor: $59.8 \%), p=0.047$. Sixty-three of the falls had no consequences The other falls resulted in 25 fractures, 10 dislocations, 14 sprains, three torn muscles, five strains, 15 serious abrasions, 93 oedemas and 128 slight scratches. Of the 158 fallers, 108 were classified as having suffered slight injury and 50 as having suffered severe injury, taking into account each participant's most severe fallrelated injury.

Fallers had poorer values than non-faller participants for almost all variables (Tables 1 and 2). The exceptions were for some physical activity (moderate, vigorous and total), body composition (body lean mass index, body mass index, waist circumference), and flexibility (lower body) variables. Fallers showed more co-morbidities (33\% more diseases and physical impairments) poorer body composition (2.7\% more body fat), and physical fitness $(8.3 \%$ lower balance), and reported living in

Table 1. Participants characteristics: age, education, co-morbidities, fear of falling, environmental hazards and physical activity according falls and fall-related injuries occurrence.

\begin{tabular}{|c|c|c|c|c|c|c|}
\hline & Part & & & Falle & & \\
\hline & Non-fallers $(n=348)$ & Fallers $(n=158)$ & $p$ Value & No/slight injury $(n=108)$ & Severe injury $(n=50)$ & $p$ Value \\
\hline Age (years) & $67.5 \pm 6.8$ & $69.6 \pm 6.6$ & 0.001 & $69.5 \pm 6.9$ & $69.8 \pm 6.2$ & 0.787 \\
\hline Education (years) & $7.0 \pm 4.2$ & $5.3 \pm 4.0$ & $<0.001$ & $5.2 \pm 3.7$ & $5.5 \pm 4.7$ & 0.764 \\
\hline Co-morbidities $(n)$ & $2.9 \pm 2.1$ & $4.3 \pm 2.6$ & $<0.001$ & $4.2 \pm 2.6$ & $4.5 \pm 2.6$ & 0.553 \\
\hline Fear of falling (point) & $1.6 \pm 2.4$ & $4.0 \pm 4.5$ & $<0.001$ & $3.4 \pm 3.8$ & $5.2 \pm 5.6$ & 0.050 \\
\hline Environmental hazards $(n)$ & $5.3 \pm 2.6$ & $6.3 \pm 2.8$ & $<0.001$ & $6.0 \pm 2.7$ & $7.0 \pm 3.1$ & 0.035 \\
\hline Walking (MET-min/week) & $778 \pm 573$ & $669 \pm 508$ & 0.039 & $635 \pm 438$ & $741 \pm 633$ & 0.225 \\
\hline Moderate-PA (MET-min/week) & $2009 \pm 1308$ & $1818 \pm 1131$ & 0.114 & $1767 \pm 1107$ & $1928 \pm 1187$ & 0.407 \\
\hline Vigorous PA (MET-min/week) & $192 \pm 637$ & $225 \pm 931$ & 0.636 & $226 \pm 1011$ & $224 \pm 739$ & 0.990 \\
\hline Total PA (MET-min/week) & $2979 \pm 1628$ & $2712 \pm 1815$ & 0.100 & $2628 \pm 1816$ & $2893 \pm 1817$ & 0.395 \\
\hline PA level (\% of participants) & & & & & & \\
\hline Low & 52.0 & 48.0 & & 50.0 & 50.0 & \\
\hline Moderate & 65.6 & 34.4 & 0.095 & 81.0 & 19.0 & 0.068 \\
\hline High & 71.0 & 29.0 & & 65.4 & 34.6 & \\
\hline Falls $(n)$ & - & $1.89 \pm 1.63$ & - & $1.7 \pm 1.5$ & $2.3 \pm 1.9$ & 0.074 \\
\hline Gender ( $\%$ of participants) & & & & & & \\
\hline Females & 66.2 & 33.8 & 0.020 & 68.9 & 31.1 & 0.722 \\
\hline Males & 77.6 & 22.4 & & 65.4 & 34.6 & \\
\hline
\end{tabular}

PA, physical activity.

Data are mean \pm standard deviation or percentage of participants in each physical activity or gender categories. 
Table 2. Participants characteristics: physical fitness according falls and fall-related injuries occurrence.

$N=506$

Participants

Fallers

Non-fallers $(n=348) \quad$ Fallers $(n=158) \quad p$ Value $\quad$ No/slight injury $(n=108) \quad$ Severe injury $(n=50) \quad p$ Value

Height $(\mathrm{cm})$

Weight $(\mathrm{kg})$

$157.5 \pm 8.6$

$69.9 \pm 12.4$

$28.1 \pm 4.3$

$17.1 \pm 2.2$

$38.7 \pm 6.2$

$91.7 \pm 11.4$

$0.43 \pm 0.15$

$17.1 \pm 4.6$

$17.7 \pm 4.5$

$-1.4 \pm 9.7$

$-7.9 \pm 10.0$

$5.5 \pm 1.2$

$529.6 \pm 87.7$

$0,12 \pm 0,58$

$33.7 \pm 4.9$

$\begin{array}{rr}154.4 \pm 7.8 & <0.001 \\ 68.9 \pm 12.3 & 0.403 \\ 28.9 \pm 4.6 & 0.085 \\ 16.8 \pm 2.2 & 0.117 \\ 41.4 \pm 5.6 & <0.001 \\ 93.2 \pm 11.4 & 0.177 \\ 0.38 \pm 0.12 & 0.001 \\ 15.6 \pm 4.5 & 0.001 \\ 16.6 \pm 4.3 & 0.009 \\ -1.6 \pm 8.7 & 0.863 \\ -11.9 \pm 11.6 & <0.001 \\ 6.0 \pm 1.8 & 0.001 \\ 495.0 \pm 90.1 & <0.001 \\ -0,16 \pm 0,65 & <0.001 \\ 30.9 \pm 6.0 & <0.001\end{array}$

$154.2 \pm .7 .9$

$68.6 \pm 12.8$

$28.83 \pm 4.81$

$16.78 \pm 2.22$

$41.3 \pm 5.3$

$93.13 \pm 12.41$

$0.38 \pm 0.12$

$15.6 \pm 4.5$

$16.1 \pm 4.3$

$-2.2 \pm 9.0$

$-12.9 \pm 11.6$

$6.1 \pm 1.9$

$493.8 \pm 87.5$

Mean fitness (SD)

Balance (point)

$30.9 \pm 6.0$

$<0.001$

$-0.21 \pm 0.66$

$30.8 \pm 5.6$

Data expressed as mean \pm standard deviation (SD).

Table 3. Predictors of falls and fall-related severe injuries.

\begin{tabular}{l}
\hline \\
\hline Falls \\
Vigorous PA (100 MET-min/week) \\
Total PA (100 MET-min/week) \\
Co-morbidities $(n)$ \\
Fear of falling (point) \\
Environmental hazards $(n)$ \\
Body lean mass index $\left(\mathrm{kg} / \mathrm{m}^{2}\right)$ \\
Body fat mass $(\%)$ \\
Balance (point) \\
Severe injury \\
Physical activity level \\
Low \\
Moderate \\
High \\
Mean fitness $(S D)$ \\
Falls $(>3)$
\end{tabular}

$$
\text { OR } 95 \% \mathrm{CI}
$$

$1.052(1.016-1.087)$

$0.982(0.967-0.998)$

$1.119(1.011-1.238)$

$1.131(1.054-1.213)$

$1.122(1.036-1.216)$

$0.914(0.846-0.987)$

$1.037(1.006-1.068)$

$0.950(0.919-0.982)$

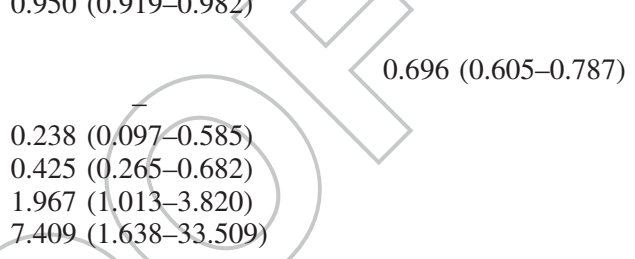

Cut-off point: 0.283

0.767

0.592

495

496

497

498

499

500

501

502

The multivariate model revealed an area under the curve (AUC) of 0.746 (CI 95\%: 0.701-0.792) with an optimal cut-off point of $0.27469(\sim 27.5 \%)$ for the probability of falling, which corresponds to a sensitivity of $74.1 \%$ and a specificity of $64.4 \%$ (Table 3 ). The equation resulting from binary logistic regression was:

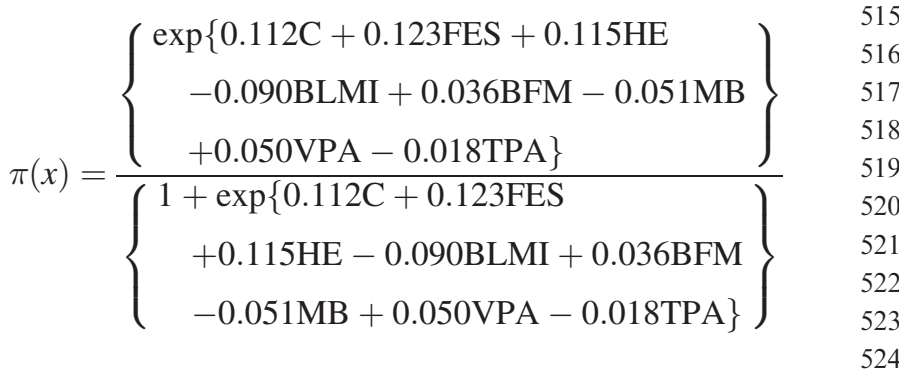

where $\pi(x)$ is the probability of falling, exp is exponential; $\mathrm{C}$ is 525 co-morbidities (number of chronic diseases and physical impair- 526 ments); FES is fear of falling score (point); HE is the number of 527 environmental hazards; BLMI is body lean mass index $\left(\mathrm{kg} / \mathrm{m}^{2}\right) ; 528$ 


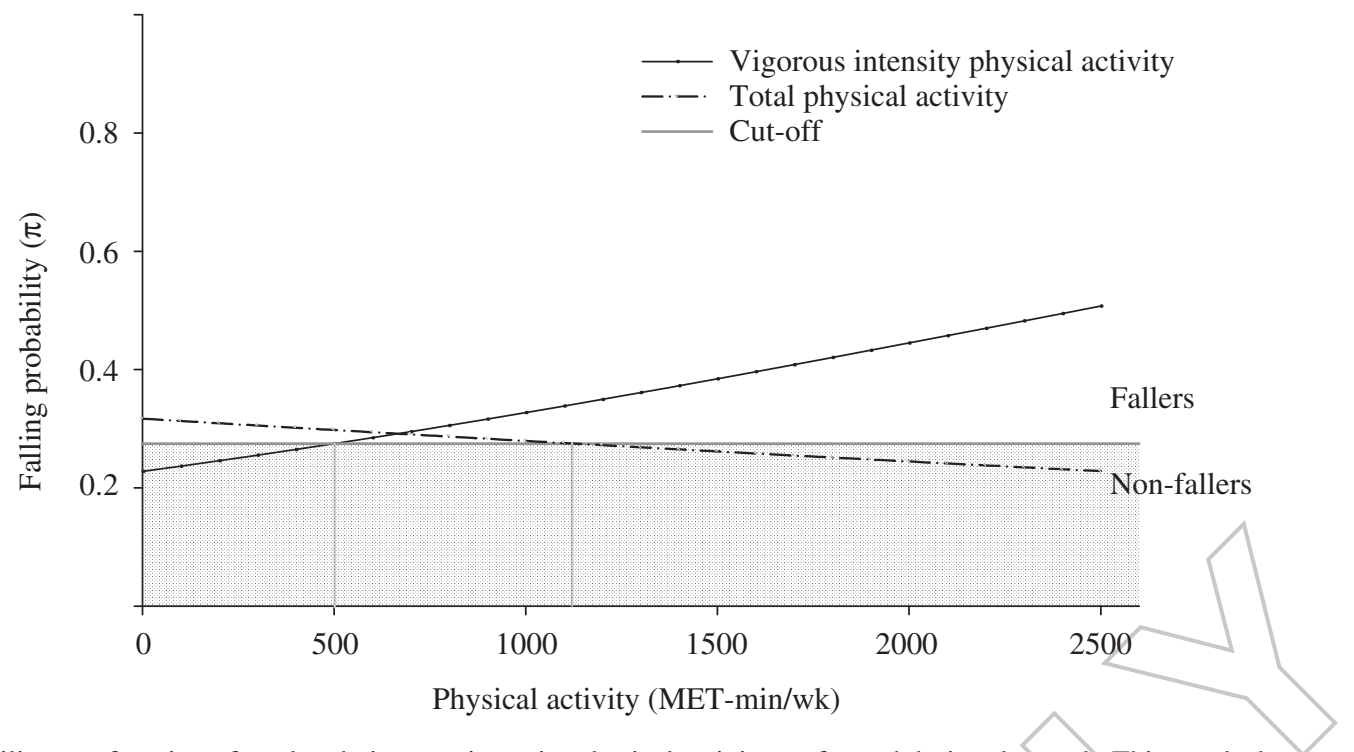

Figure 1. Fall probability as a function of total and vigorous-intensity physical activity performed during the week. This graph shows cut-off values for total and for vigorous-intensity physical activity (1125 MET-min/week and $501 \mathrm{MET}$-min/week, respectively) that discriminate fallers from non-fallers.

BFM is body fat mass (\%); $\mathrm{MB}$ is balance (multidimensional) score (point); VPA is vigorous-intensity physical activity performed during the week (100 MET-min/week) and TPA is total physical activity performed during the week (100 MET$\mathrm{min} /$ week).

As described in statistical analysis, the equation was solved separately for each of the significant physical activity variables (total and vigorous physical activity), using the identified cut-off point for falls probability. To this end, the 50th percentile value was specified for all variables in the equation except for total physical activity (in the first calculation) and vigorous physical activity (in the second calculation). The 50th percentile corresponded to three diseases or physical impairments in co-morbidities; one point in fear of falling; five environmental hazards; $16.9 \mathrm{~kg} / \mathrm{m}^{2}$ in body lean mass index; $40.3 \%$ in body fat mass; 34 points in balance; 0 MET-min/week in vigorous physieal activity; 2514 MET-min/week in total/physical activity. Figure 1 shows the probability of falling as a function of physical activity (point estimation). An increase in the probability of falling was observed with the increase in vigorous physical activity, and a decrease in the probability of falling with the increase in total physical activity. Regarding the cut-off point $(0.27469)$, fallers were identified as those who performed more than 501 MET-min/ week of vigorous physical activity and less than 1125 MET-min/ week of total physical activity (Figure 1).

\section{Fall-related injuries occurrence}

Multivariate regression analysis showed that the significant predictors for fall-related severe injuries in fallers $(n: 158)$ were fitness, falling more than three times, and physical activity level (Table 3). The Hosmer and Lemeshow goodness-of-fit test was not significant $(p=0.662)$ and the AUC was 0.696 (CI 95\%: $0.605-0.787)$. The likelihood of the occurrence of severe fallrelated injuries increased $96.8 \%$ for each additional standard deviation of mean fitness. For those who fell more than three times, the likelihood of severe injury was almost 7.5 times higher. Finally, multivariate regression analysis showed that the likelihood of the occurrence of severe fall-related injuries for those classified as moderate physical activity level decreases $76.2 \%$ relative to those classified as low physical activity level, and $57.5 \%$ for those classified as high physical activity level relative to those classified as low level (Figure 2).

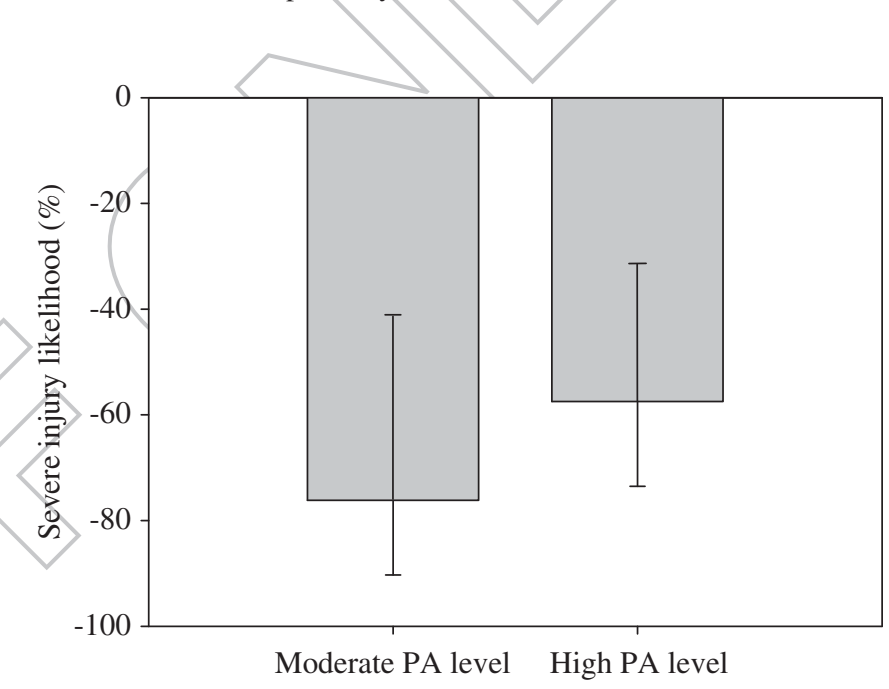

Figure 2. Severe injury likelihood according to physical activity (PA) level. This graph quantifies the decrease in the likelihood of fall-related severe injuries in fallers with moderate (less 76\%) and high (less 58\%) physical activity levels relative to fallers with low-physical activity level (considered as reference).

\section{Discussion}

The main purpose of this study was to analyse the role of physical activity (type, level and amount) in falls and related injuries, after adjustment for physical fitness, fear of falling, and environmental hazards. Contrary to some beliefs $[14,16]$, the results showed that the total amount of physical activity did not promote the occurrence of falls and injuries. Increasing $500(5 \times 100)$ MET$\mathrm{min} /$ week in total physical activity (i.e. $\sim 90 \mathrm{~min}$ on moderate physical activity +40 min on walking) was shown to lead directly to a decrease in the likelihood of falling by $8.6 \%$. As total physical activity contributes to improve body composition and balance [13], this it would lead indirectly to a further decrease in the risk of falling: $4.4 \%$ less by increasing $0.5 \mathrm{~kg} / \mathrm{m}^{2}$ in body lean mass index, $11.4 \%$ less by decreasing $3 \%$ in body fat mass $(\sim 2.1 \mathrm{~kg}$ in men and $\sim 2.4 \mathrm{~kg}$ in women), and $13.4 \%$ less by increasing three points in balance. That is, it would lead indirectly to a decrease in the likelihood of falling by an additional $30 \%$. However, the probability of falling increased with vigorous physical activity. Thus, according IPAQ criteria, total physical activity of at least 1125 MET-min/week with no more than 501 MET-min/week (i.e. 
661 less than $\sim 1 \mathrm{~h} /$ week) of vigorous physical activity significantly 662 reduced falls. This total physical activity amount is more than 663 double the lowest value recommended for older people for general 664 health benefits (i.e. $150 \mathrm{~min} /$ week $\times 3 \mathrm{MET}$ : $450 \mathrm{MET}-\mathrm{min} /$ week) 665 of moderate intensity $[13,23,35]$. Furthermore, physical activity 666 also seems to influence fall-related injuries. Being active, 667 particularly at a moderate level, was shown to significantly 668 decrease the occurrence of severe fall-related injuries in fallers.

669 Total physical activity reduces fall probability because it 670 improves the individual's capacity to perform tasks [14], even 671 in more frail people, with lower levels of health (high 672 co-morbidities), body composition and balance and with a high 673 fear of falling score. In this study, independently of the other 674 predictors, an increase of $100 \mathrm{MET}-\mathrm{min} /$ week in total physical 675 activity, measured by IPAQ, led to a $2 \%$ decrease in the risk of one 676 or more falls. Peeters et al. [36] reported a 4\% decrease in the risk 677 of recurrent falling for each $100 \mathrm{MET}-\mathrm{min} /$ week in total physical 678 activity measured by using the LASA Physical Activity 679 Questionnaire. Vigorous physical activity reduced the risk of 680 falls by increasing total activity, but only up to the threshold 681 defined above (501 MET-min/week). Above this threshold, 682 vigorous physical activity only increased the risk of falls (5\% 683 for each $100 \mathrm{MET}-\mathrm{min} /$ week); this is most likely because at a 684 higher physical activity levels (e.g. farming), tasks are more 685 demanding [4] and the propensity for falls is greater. This 686 may help to explain why either inactivity or excessive physical 687 activity promoted an increase of reported falls in other studies, $688[16,17]$ particularly when heavy physical tasks were involved, like 689 sports [18].

690 Being active was shown to chiefly decrease the risk of severe 691 fall-related injuries, possibly because falls occurred less often (i.e. 692 the chance of injury is reduced), and/or resistance to fall impact 693 may be higher [37]. In this study, a positive association was 694 observed between the number of falls and the occurrence of fall695 related injuries. In particular, it was found that falling more than 696 three times a year exponentially increased the risk of severe 697 injury. Old people that were high active were less prone to fall698 related injury than those who were most inactive. However, the moderately active participants had the lowest risk. Most of the studies on fall-related injuries focus on fractures and point to bone mineral density as a major risk factor, recommending regular exercise as a preventive measure [38]. Only a few/researchers have begun to focus on other fall-related injuries and physical activity. A study conducted by Gill et al. [19] found a positive association between injurious falls and lower subsequent household physical activity besides a negative association between injurious falls and higher recreational physical activity.

In general, good fitness appears to reduce the severity of fallrelated injuries [39], probably because fit subjects react during the fall, thereby reducing the impact. On the other hand, for fallers, a higher level of fitness may contribute to more severe fall-related injuries since it has been observed that high-fitness participants were more confident and put themselves in situations that are more risky. A negative association was observed between the average score of fitness and fear of falling ( $r:-339, p<0.001$, $n=506)$ and a positive association between the average score of fitness and the number of falls when performing high-difficulty tasks $(r: 0.179 ; p=0.033, n=158)$. This may explain why in the present study a high level of fitness, especially high upper body strength, although decreasing the probability of the occurrence of falls, appeared to be a risk factor for severe injury in fallers.

The main intrinsic fall risk factors affecting an individual's ability to perform physical tasks successfully (without falling) were fear of falling (OR: 13.1\%), co-morbidities (OR: 11.9\%), body fat (OR: $3.7 \%$ ) and lean mass (OR 8.6\%), and balance (OR: $5.0 \%)$. Fear of falling has been considered a risk factor for falling and consequent injuries [30,40]. However, in this study, it was 727 observed that people with greater fear of falling avoided daily 728 tasks involving greater risk, like going up and down stairs or 729 getting objects out of cupboards and wardrobes. Moreover, they 730 also performed less total physical activity $(r:-0.144, p<0.001,731$ $n=506)$, had reduced physical fitness $(r$ : -0.181 to $-0.380,732$ $p<0.001, n=506)$ and had more diseases and physical impair- 733 ments $(r: 0.434, p<0.001, n=506)$, besides falling more and 734 suffering more severe injuries, as also noted by others [11,12]. 735 These results suggest that fear of falling works mostly as a self- 736 perceived risk of falling due to frailty and previous experience of 737 falling and injury (a protective mechanism) and less as a real risk 738 factor for falling and consequent injury. (3),31]. Data from multivariate regression analysis revealed, nevertheless, that fall probability is reduced when older people are healthier and fitter and they do not fall as often as younger less healthier and unfit persons, even when living in environments that are more hazardous. It appears that it is reduced co-morbidities and good physical fitness that sustains the ability to perform more demanding tasks without falling. In fact, extrinsic risk factors (environment) appear to contribute to falls, particularly in the presence of intrinsic risk factors (co-morbidities, decreased balance and poor body composition), independently of age. This is in accordance with the recommendation of Nitz and Choy [9] for prevention interventions in middle-age as well as in old age.

Results regarding physical activity were adjusted for the selected main risk factors for falls and consequent injuries. However, an increment in the amount of physical activity can promote improvements in physical fitness [13]. In practice, besides a direct effect (isolated) of physical activity on the probability of falling and injury, there may be an indirect effect (additional) on the occurrence of these events associated with the changes induced by physical activity on the other predictors [13]. This additional benefit depends on the amount of change induced by physical activity on each predictor. For example, the number of co-morbidities varied between 0 and 12 (a difference of 12 units), and for an increment of 1 unit, the OR was 1.119; but the percentage of body fat mass varied from $10.2 \%$ to $55.7 \%$ (a difference of 45.5 units) and for an increment of 1 unit, the OR was 1.037. Thus, despite a low OR magnitude, fat mass may have a higher impact on falls than co-morbidities, depending on the amount of variation (one unit in co-morbidities corresponds to $8.3 \%$ of co-morbidities variation, while 1 unit in body fat mass corresponds to $2.2 \%$ of body fat mass variation).

The participants in this study were physically and socially active, maintaining average levels of capacities and independence [5]. Even the eldest participants reported, in the interview, looking after themselves, housekeeping, going out for shopping, performing exercise and performing other advanced daily activities. This might explain some inconsistency with results from other studies in which aged and frail people showed a higher risk for indoor falls than outdoor falls [41]. In the present study, there was a higher proportion of outdoor falls compared to indoor falls in aged persons and a low proportion of outdoor falls compared to indoor falls in middle-aged persons. In contrast to the findings of other researchers [1,42], no differences were observed between younger (middle-aged) and older (aged) people regarding the amount of physical activity reported (nevertheless there was a negative correlation between the amount of total physical activity and age $(r:-0.118, p=0.008)$. If frail persons stay at home, there will be the chance of indoors falls. If these persons go outside, the chance of outdoor falls will be greater, most likely because outdoor hazards and task demands are higher than indoor hazards and task demands and thus exposure risk will be higher [14]. 29 0 2 33 5 36 737
738 
Limitations of this study include the sample size regarding

those who fell and suffered injuries (and therefore the statistical power of the model for predicting the severity of fall-related injuries), and the relatively small number of men compared with women since some authors point to gender as a moderator of falls and consequent injuries $[1,43]$. The use of the number of chronic diseases and physical impairments for analysing health status influence may be questionable because a disease such as epilepsy obviously predisposes individuals to falls more than asthma. However, the significance of each disease and physical impairment in the regression analysis was tested and "co-morbidities" was the variable that best explained the occurrence of falls. This methodology has already been used in similar studies [9]. Additionally, the use of a retrospective recall was considered acceptable for assessing falls and injuries occurring during the last 12 months, given that a normal cognitive function was required for all participants and the circumstances surrounding each fall and its consequences were described in detail. Still, it is possible that there was an underestimation of the number of falls that occurred in this period. Unlike other works that focused exclusively on recurrent fallers [30], the present study includes one-time fallers, also showing a similar capacity for discriminating fallers (AUC: 0.746 , CI 95\%: 0.701-0.792). Furthermore, the predicted probabilities that were generated by the cross-validation procedure had a similar AUC (0.723, 95\% CI: $0.675-0.771)$ and the results were similar when a Poison regression was performed using the number of falls as the dependent variable. A major limitation of this investigation was the evaluation of physical activity by means of questionnaire because the use of this method tends to lead to an overestimation in amounts of physical activity [44]. Moreover, the relationship between current physical activity and the retrospective reports of falls and injuries was explored cross-sectionally. However, the inherent error associated with this design has been minimised because participants reported maintaining their physical activity over the past year, as well as their health status, weight and environmental hazards. Furthermore, subjects that suffer from recent illnesses or disabilities resulting in a temporary loss of physical function were excluded from the study. These aspects and the fact that reported co-morbidities, particularly chronic diseases such as diabetes, or physical impairments such as poor vision are very unlikely to result from falls, suggest a causal relationship between the predictors of falls and fall-related injuries (including physical activity) and the occurrence of these events. However, the relationship between variables may be interpreted bi-directionally in a cross-sectional study.

\section{Conclusion}

Increasing total physical activity does not promote falls whereas vigorous physical activity exceeding 500 MET-min/week seems to increase the occurrence of falls. Being sufficiently active reduces fall-related injuries by reducing falls and by safeguarding against severe injury when falls occur. Reduced co-morbidities and a good level of physical fitness, principally balance, improve the ability to perform physical tasks without falling, even in environments that are more hazardous. Nevertheless, for fallers, a good level of physical fitness may not safeguard against severe fall-related injuries. Thus, for independent adults aged over 50 years, at least $1125 \mathrm{MET}-\mathrm{min} /$ week of total physical activity with equal or lower than 500 MET-min/week of vigorous physical activity appears to reduce falls and, therefore, fall-related injuries.

\section{Acknowledgements}

The authors would like to thank all the volunteers, who participated in this study, and Joana Vazquez and Daniel Dolan for reviewing the text in English.

\section{Declaration of interest}

The authors declare that there is no conflict of interest.

Portuguese Science and Technology Foundation $(\mathrm{PhD}$ grant SFRH/BD/31450/2006) provided financial support to conduct this study. The sponsors had no role in the design, analysis, or preparation of this manuscript.

\section{References}

1. Etman A, Wijlhuizen GJ, van Heuvelen MJ, et al. Falls incidence underestimates the risk of fall-related injuries in older age groups: a comparison with the FARE (Falls risk by Exposure). Age Ageing 2012;41:190-5

2. Tucker MG, Kavanagh JJ, Morrison S, Barrett RS. What are the relations between voluntary postural sway measures and falls-history status in community-dwelling older adults? Arch Phys Med Rehabil 2010;91:750-8.

3. St George RJ, Fitzpatrick RC, Rogers MW, Lord SR. Choice stepping response and transfer times: effects of age, fall risk, and secondary tasks. J Gerontol A Biol Sci Med Sci 2007;62:537-42.

4. Glazier PS, Davids K. Constraints on the complete optimization of human motion. Sports Med 2009;39:15-28.

5. Rikli R, Jones CJ. Functional fitness normative scores for community-residing older adults. J Aging Phys Act 1999;7:160-79.

6. de Rekeneire N, Visser M, Peila R, et al. Is a fall just a fall: correlates of falling in healthy older persons. The health, aging and body composition study. J Am Geriatr Soc 2003;51:841-6.

7. WHO. 2011 World Health Statistics 2011. Available from: http:// www.who.int/whosis/whostat/EN_WHS2011_Full.pdf [last accessed 12 Feb 2012].

8. Kannus P, Niemi S, Palvanem M, Parkkari J. Rising incidence of fall-induced injuries among elderly adults. J Publ Health 2005;13 212-15.

9. Nitz JC, Choy NL. Falling is not just for older women: support for pre-emptive prevention intervention before 60 . Climacteric $2008 ; 11$ : 461-6.

10. Li W, Keegan TH, Sternfeld B, et al. Outdoor falls among middleaged and older adults: a neglected public health problem. Am J Publ Health 2006;96:1192-200.

11. Boyd R, Stevens JA. Falls and fear of falling: burden, beliefs and behaviours. Age Ageing 2009;38:423-8.

12. Gaxatte C, Nguyen T, Chourabi F, et al. Fear of falling as seen in the Multidisciplinary falls consultation. Ann Phys Rehabil Med 2011 $54: 248-58$.

13. Chodzko-Zajko WJ, Proctor DN, Fiatarone Singh MA, et al. American college of sports medicine position stand. Exercise and physical activity for older adults. Med Sci Sports Exerc 2009;41: 1510-30.

14. Wijlhuizen GJ, Chorus AM, Hopman-Rock M. The FARE: a new way to express FAlls Risk among older persons including physical activity as a measure of exposure. Prev Med 2010;50:143-7.

15. Sherrington C, Whitney JC, Lord SR, et al. Effective exercise for the prevention of falls: a systematic review and meta-analysis. J Am Geriatr Soc 2008;56:2234-43.

16. Chan BK, Marshall LM, Winters KM, et al. Incident fall risk and physical activity and physical performance among older men: the Osteoporotic Fractures in Men Study. Am J Epidemiol 2007;165 696-703.

17. Gregg EW, Cauley JA, Seeley DG, et al. Physical activity and osteoporotic fracture risk in older women. Study of Osteoporotic Fractures Research Group. Ann Intern Med 1998;129:81-8.

18. Peeters GM, Verweij LM, van Schoor NM, et al. Which types of activities are associated with risk of recurrent falling in older persons? J Gerontol A Biol Sci Med Sci 2010;65:743-50.

19. Gill DP, Zou GY, Jones GR, Speechley M. Injurious falls are associated with lower household but higher recreational physical activities in community-dwelling older male veterans. Gerontology 2008;54:106-15.

20. Choi YS, Lawler E, Boenecke CA, et al. Developing a multisystemic fall prevention model, incorporating the physical environment, the care process and technology: a systematic review. J Adv Nurs 2011;67:2501-24.

21. Aoyama M, Suzuki Y, Onishi J, Kuzuya M. Physical and functional factors in activities of daily living that predict falls in communitydwelling older women. Geriatr Gerontol Int 2011;11:348-57. 
22. Folstein MF, Folstein SE, McHugh PR. "Mini-mental state". A practical method for grading the cognitive state of patients for the clinician. J Psychiatr Res 1975;12:189-98.

23. Craig CL, Marshall AL, Sjostrom M, et al. International physical activity questionnaire: 12-country reliability and validity. Med Sci Sports Exerc 2003;35:1381-95.

24. Gill DP, Zou GY, Jones GR, Speechley M. Comparison of regression models for the analysis of fall risk factors in older veterans. Ann Epidemiol 2009;19:523-30.

25. Rikli R, Jones CJ. Development and validation of a functional fitness test for community-residing older adults. J Aging Phys Act 1999;7:129-61.

26. Owsley C, Sloane M, McGwin Jr G, Ball K. Timed instrumental activities of daily living tasks: relationship to cognitive function and everyday performance assessments in older adults. Gerontology 2002;48:254-65.

27. Rose DJ, Lucchese N, Wiersma LD. Development of a multidimensional balance scale for use with functionally independent older adults. Arch Phys Med Rehabil 2006;87:1478-85.

28. Zaluska W, Malecka T, Mozul S, Ksiazek A. [Whole body versus segmental bioimpedance measurements (BIS) of electrical resistance $(\mathrm{Re})$ and extracellular volume (ECV) for assessment of dry weight in end-stage renal patients treated by hemodialysis]. Przegl Lek 2004; 61:70-3.

29. Tinetti M, Richman D, Powell L. Falls efficacy as a measure of fear of falling. J Gerontol 1990;45:P239-43.

30. Pluijm SM, Smit JH, Tromp EA, et al. A risk profile for identifying community-dwelling elderly with a high risk of recurrent falling: results of a 3-year prospective study. Osteoporos Int 2006;17: 417-25.

31. Tinetti ME, Speechley M, Ginter SF. Risk factors for falls among elderly persons living in the community. N Engl J Med 1988;319: 1701-7.

32. Hosmer D, Lemeshow S. Applied logistic regression. 2nd ed. New York: Wiley; 2000.
33. Scholtissen S, Guillemin F, Bruyere O, et al. Assessment of 991 determinants for osteoporosis in elderly men. Osteoporos Int 2008; 20:1157-66.

34. Efron B, Tibshirani R. An introduction to the bootstrap. Boca Raton (IL): Chapman and Hall; 1993.

35. Kesaniemi A, Riddoch CJ, Reeder B, et al. Advancing the future of physical activity guidelines in Canada: an independent expert panel interpretation of the evidence. Int J Behav Nutr Phys Act 2010;7:41.

36. Peeters GM, van Schoor NM, Pluijm SM, et al. Is there a U-shape association between physical activity and falling in older persons? Osteoporos Int 2010;21:1189-95.

37. Rose DJ. Preventing falls among older adults: no "one size suits all' intervention strategy. J Rehabil Res Dev 2008;45:1153-66.

38. Kannus P, Sievanen H, Palvanen M, et al. Prevention of falls and consequent injuries in elderly people. Lancet 2005;366: 1885-93.

39. Bergland A, Wyller TB. Risk factors for serious fall related injury in elderly women living at home. Inj Prev 2004;10:308-13.

40. Lloyd BD, Williamson DA, Singh NA, et al. Recurrent and injurious falls in the year following hip fracture: a prospective study of incidence and risk factors from the Sarcopenia and Hip Fracture study. J Gerontol A Biol Sci Med Sci 2009;64:599-609.

41. Kelsey JL, Berry SD, Procter-Gray E, et al. Indoor and outdoor falls in older adults are different: the maintenance of balance, independent living, intellect, and Zest in the Elderly of Boston Study. J Am Geriatr Soc 2010;58:2135-41.

42. Mertz KJ, Lee DC, Sui X, et al. Falls among adults: the association of cardiorespiratory fitness and physical activity with walkingrelated falls. Am J Prev Med 2010;39:15-24.

43. Yeo YY, Lee SK, Lim CY, et al. A review of elderly injuries seen in a Singapore emergency department. Singapore Med J 2009;50: 278-83.

44. Welk G. Physical activity assessments for health-related research. Champaign (IL): Human Kinetics; 2002. 